\title{
Presynaptic Actions of Carbachol and Adenosine on Corticostriatal Synaptic Transmission Studied in vitro
}

\author{
Robert C. Malenka ${ }^{1}$ and Jeffery D. Kocsis ${ }^{2}$ \\ 'Department of Psychiatry and Behavioral Sciences, Stanford University School of Medicine, Stanford, California 94305 \\ and Veterans Administration Medical Center, Palo Alto, California 94304, and ${ }^{2}$ Department of Neurology, Yale University \\ Medical School, New Haven, Connecticut 06410
}

The purpose of this study was to identify, in the in vitro rat neostriatal slice preparation, an electrophysiological response corresponding to the activation of striatal neurons by cortical afferents, and to study the actions of a variety of putative neurotransmitters on modulating synaptic transmission at this synapse. Local stimulation of the neostriatal slice evokes a field potential composed of 2 prominent negativities. Experiments using calcium antagonists and tetrodotoxin indicate that the first negativity $(\mathrm{N} 1)$ reflects the direct activation of intrinsic neurons and axons, while the second negativity (N2) is synaptically mediated. The afferent fibers eliciting the second negativity have not previously been identified because of the mixing of afferents within the striatum. However, similar field potential responses are elicited by stimulation of the corpus callosum, in which the only striatal afferents are crossed corticostriatal fibers. Kynurenate, $\gamma$-D-glutamylglycine, and piperidine dicarboxylate all reversibly reduced or abolished N2, suggesting that the transmitter generating the response is an excitatory amino acid. Together, these results strongly suggest that $\mathrm{N} 2$ reflects activation of striatal neurons by corticostriatal afferents.

Three putative striatal neurotransmitters, dopamine, acetylcholine and adenosine, were studied with respect to their ability to modulate the corticostriatal response. Dopamine had minimal effects on the waveform of the field potential. In contrast, carbachol or adenosine consistently reversibly reduced or eliminated N2. Atropine blocked carbachol's actions, while theophylline blocked adenosine's actions, indicating that the compounds were acting on muscarinic and adenosine receptors, respectively. To test whether these were primarily pre- or postsynaptic actions, we recorded the response to ionophoretically applied glutamate while simultaneously recording the synaptically evoked field potential from the same location. Kynurenate reduced both responses simultaneously, while carbachol or adenosine reduced only the synaptically mediated N2. From these results we propose that the terminals of corticostriatal afferents may possess muscarinic and adenosine receptors that function to inhibit transmitter release.

\footnotetext{
Received Sept. 3, 1987; revised Jan. 22, 1988; accepted Jan. 29, 1988.

This work was supported by grants from the Medical Research Service of the Veterans Administration and N.I.H.

Correspondence should be addressed to Robert C. Malenka, M.D., Ph.D., Department of Psychiatry and Behaviorial Sciences, Stanford University School of Medicine, Stanford, CA 94305

Copyright (C) 1988 Society for Neuroscience $0270-6474 / 88 / 103750-07 \$ 02.00 / 0$
}

The mammalian neostriatum contains a variety of putative neurotransmitters, including the highest concentrations of acetylcholine and dopamine found among any structures in the mammalian forebrain (Graybiel and Ragsdale, 1983). It thus has been the subject of much research aimed at elucidating its biochemical anatomy. Electrophysiological analysis has proven more difficult, primarily because of its nonlaminar organization and the limitations inherent in utilizing in vivo preparations. Recently, however, several investigators have begun examining the electrophysiological properties of striatal neurons using the in vitro striatal slice preparation (Misgeld et al., 1979; Kita et al., 1985; Cordingley and Weight, 1986).

The purpose of this study was to identify, in the slice preparation, the electrophysiological response generated by the cortical input to the neostriatum and to examine the actions of compounds that may presynaptically inhibit this input. The entire cerebral cortex projects topographically to the neostriatum and thereby provides a major afferent input (Kemp and Powell, 1971). Presynaptic modulation of the corticostriatal pathway would have profound consequences for the transfer of information from cortex to striatum and for the functioning of the extrapyramidal, as well as other behaviorally relevant, systcms.

Yamamoto (1973) first reported that local stimulation in the striatal slice evokes a field potential consisting of 2 negative waves. It has been suggested that the first component represents the direct and antidromic activation of axons and intrinsic neurons, while the second component appears to be synaptically mediated (Takagi and Yamamoto, 1978; Misgeld et al., 1979; Cordingley and Weight, 1986). The identity of the neurotransmitter responsible for generating the second negativity is unclear. Misgeld and his colleagues (1980) have reported that nicotinic antagonists block this component, and therefore concluded that it is mediated by $\mathrm{ACh}$ released from intrinsic cholinergic neurons and acting on nicotinic receptors. On the other hand, Cordingley and Weight (1986) have found that nicotinic antagonists have no effect on the field potential's second component and instead have proposed that it is mediated by a glutamatelike transmitter. In the initial part of this study, we examine the actions of both nicotinic antagonists and excitatory amino acid antagonists on the field potential evoked by local stimulation in the in vitro rat neostriatal slice preparation. In addition, by carefully positioning stimulating and recording microelectrodes, we attempt to unequivocally identify a corticostriatal response.

Several compounds may have significant actions on the cortical input to the neostriatum, specifically, dopamine (DA), carbachol, and adenosine. It has been suggested that DA receptors 
A

A CONTROL

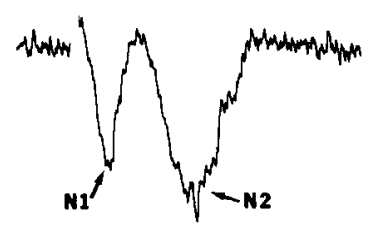

D KYNURENATE

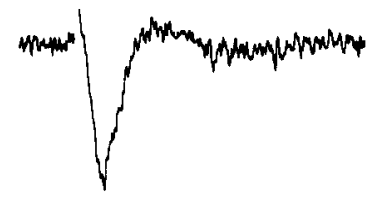

B $0.5 \mathrm{Ca}, 6 \mathrm{Mg}$

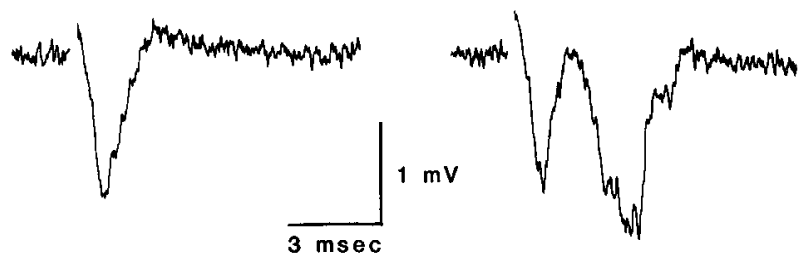

E

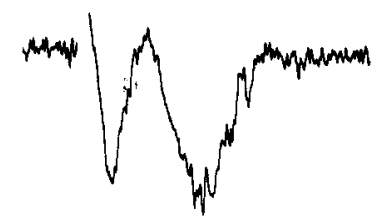

\section{WASH}
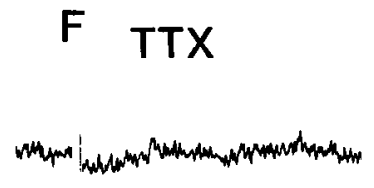

Figure 1. $\quad \mathrm{N} 2$ is synaptically mediated and is blocked by kynurenate. $A-C$, Application for $3 \mathrm{~min}$ of a solution containing $0.5 \mathrm{mM} \mathrm{CaCl}_{2}$ and $6.0 \mathrm{mM} \mathrm{MgCl}$ reversibly blocked $\mathrm{N} 2$ without affecting N1. $C-E$, Kynurenate $(1 \mathrm{~mm})$ also reversibly blocked $\mathrm{N} 2$ within 2 min of application. F, TTX $(0.3 \mu \mathrm{M})$ blocked both $\mathrm{N} 1$ and N2. In this and all subsequent experiments, a stable control field potential was obtained and drugs were then sequentially applied to the same slice. are found on the terminals of the cortical afferents to the striatum (Schwarcz et al., 1978; Kornhuber and Kornhuber, 1986) and that DA can inhibit the release of glutamate from striatal slices (Mitchell and Doggett, 1980; Rowlands and Roberts, 1980). Muscarinic agonists, such as carbachol, have been reported to decrease the second negativity of the field potential, perhaps by acting at a presynaptic locus (Takagi and Yamamoto, 1978; Weiler et al., 1984). Finally, adenosine and its receptors are found in reasonably high concentrations in the striatum (Goodman and Snyder, 1982) and in a variety of other systems have been shown to presynaptically inhibit excitatory synaptic transmission (Ginsborg and Hirst, 1972; Okada and Ozawa, 1980; Kocsis et al., 1984; Dunwiddie and Haas, 1985). In the second part of this study, we examine the actions of DA, carbachol, and adenosine on the corticostriatal response.

\section{Materials and Methods}

Neostriatal slices were prepared from female, Wistar rats (180-240 gm) using standard techniques. The animals were deeply anesthetized with sodium pentobarbital $(60 \mathrm{mg} / \mathrm{kg}$, i.p.) and decapitated. The dorsal surface of the brain was immediately exposed, and a 4-6 mm coronal section of one hemisphere was removed by hemisecting the brain and then making coronal cuts approximately 3 and $7-9 \mathrm{~mm}$ from the tip of the frontal poles. This tissue block was immediately immersed in cold $\left(2-4^{\circ} \mathrm{C}\right)$, modified Krebs solution and then glued to the stage of a Vibratome with cyanoacrylate. Coronal slices, $400 \mu \mathrm{m}$ thick, were then cut, and included neocortex, corpus callosum, and neostriatum. Slices were stored in oxygenated modified Krebs solution at room temperature and were allowed to stabilize for at least $1 \mathrm{hr}$ before being transferred to the recording chamber. A single slice was transported to the recording chamber (volume, $1.0 \mathrm{~cm}^{3}$ ), where it was held between 2 nylon nets, submerged beneath continuously flowing $\left(3-5 \mathrm{~cm}^{3} / \mathrm{min}\right)$ modified Krebs solution consisting of (in $\mathrm{mM}$ ) $\mathrm{NaCl}, 124 ; \mathrm{KCl}, 3.0 ; \mathrm{MgCl}_{2}, 2.0 ; \mathrm{CaCl}_{2}$, 2.0; $\mathrm{NaHCO}_{3}, 26.0 ; \mathrm{NaH}_{2} \mathrm{PO}_{4}, 1.3$; dextrose, 10.0. The solution was saturated with $95 \% \mathrm{O}_{2}$ and $5 \% \mathrm{CO}_{2}(\mathrm{pH} 7.4)$. All drugs were obtained from Sigma and were made up at high concentrations (10-100 $\mathrm{mM}$ ) in distilled water and diluted to their final concentrations in the bathing medium immediately before application.

Standard field potential recording techniques were used. Electrodes (2-8 M $\Omega$ ) were pulled from "omega dot" glass capillary tubing $(2.0 \mathrm{~mm}$ O.D., $1.0 \mathrm{~mm}$ I.D.) and filled with $3 \mathrm{M} \mathrm{NaCl}$. Signals were amplified using an Axon Instruments Axoprobe-1 and then digitized with a Nicolet 1170 signal averager and stored on magnetic tape. Slices were stimulated by constant-current cathodal pulses ( $40 \mu \mathrm{sec}$ duration) delivered through monopolar tungsten electrodes. The stimulating and recording microelectrodes were positioned approximately $0.5-3.0 \mathrm{~mm}$ apart, with the stimulating microelectrode placed either directly in the corpus callosum or immediately (within $500 \mu \mathrm{m}$ ) ventral to it and the recording microelectrode placed ventral to the stimulating electrode. Sodium glutamate $(1.0 \mathrm{M}, \mathrm{pH})$ was applied ionophoretically by positioning the ionophoretic microelectrode as close as possible to the recording microelectrode under visual control and then moving it through the depth of the slice to obtain a maximal response. At the conclusion of each experiment involving glutamate ionophoresis, the ionophoretic microelectrode was raised out of the slice so that its tip was in the bathing medium and the electrical response to glutamate application was then recorded. The coupling artifact thus recorded was digitally subtracted from the previously recorded glutamate responses.

\section{Results}

The field potential elicited by intrastriatal stimulation consists of 2 negativities (N1 and N2; Fig. 1A) (Yamamoto, 1973; Misgeld et al., 1979; Cordingley and Weight, 1986). N1 is thought to reflect direct activation of neuronal elements, while N2 is synaptically mediated (Takagi and Yamamoto, 1978; Misgeld et al., 1979; Cordingley and Weight, 1986). However, there is some question about the transmitter and pathway mediating N2 (Takagi and Yamamoto, 1978; Misgeld et al., 1980; Cordingley and Weight, 1986). Figure $1, A-C$, shows that $\mathrm{N} 2$ is reversibly blocked by a low-calcium $(0.5 \mathrm{~mm})$, high-magnesium $(6.0 \mathrm{~mm})$ solution, while $\mathrm{N} 1$ is unaffected $(n=5)$. The nicotinic antagonists mecamylamine (100 $\mu \mathrm{M} ; n=3)$ and $d$-tubocurarine (30 $\mu \mathrm{M} ; n=4$ ) had no effect on either component of the field potential, even when applied for up to $30 \mathrm{~min}$. In contrast, kynurenate $(1 \mathrm{~mm})$, an excitatory amino acid antagonist (Ganong et al., 1983; Jahr and Yoshioka, 1986), reversibly blocked or reduced N2 within 2 min of bath application without affecting $\mathrm{N} 1(n=21$; Fig. $1, C-E)$. Both $\mathrm{N} 1$ and N2 were blocked by TTX ( $n=3$; Fig. $1 F)$, indicating that they are both dependent on sodium-mediated spike discharges.

As would be expected, N1 was virtually unaffected by any dose of kynurenate at any stimulation strength (Fig. 2A). The antagonistic action of kynurenate on $\mathrm{N} 2$ was dose-dependent and observed at all stimulation strengths (Fig. 2B). To further test whether N2 is mediated by an excitatory amino acid, the effects of 2 other excitatory amino acid antagonists were examined. Both $\gamma$-D-glutamylglycine $(1 \mathrm{~mm} ; n=3)$ and piperidine dicarboxylate ( $1 \mathrm{mM} ; n=3$; Fig. $6 F$ ) reversibly reduced or blocked N2 without affecting N1. 

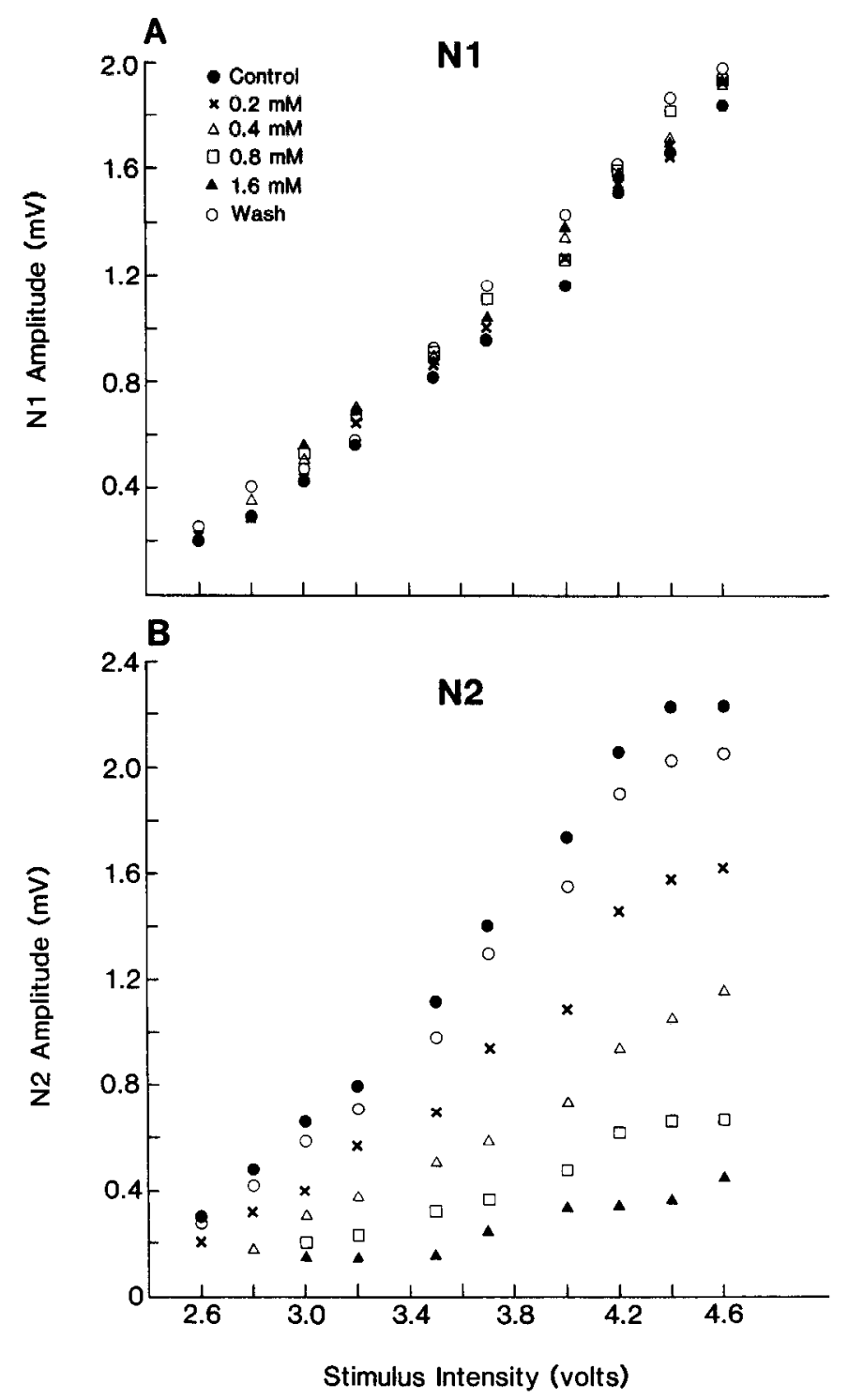

Figure 2. Kynurenate reduces N2 in a dose-dependent manner independent of stimulus intensity, while $\mathrm{N} 1$ is unaffected. The graphs show the amplitudes of $\mathrm{N} 1(A)$ and $\mathrm{N} 2(B)$ as a function of stimulation intensity from an experiment in which 4 different concentrations of kynurenate $(0.2,0.4,0.8$, and $1.6 \mathrm{~mm})$ were applied successively to the same slice. Note that after the washout of $1.6 \mathrm{~mm}$ kynurenate, the N2 amplitude returned to its control values.

It is generally accepted that the major glutaminergic input to the neostriatum derives from cortical afferents (Dray, 1980; Graybiel and Ragsdale, 1983). Therefore, it is likely, as suggested previously (Cordingley and Weight, 1986), that N2 is due to the activation of cortical afferents and the subsequent release of glutamate. Consistent with this hypothesis was our finding that we could elicit a kynurenate-sensitive N2 component with the stimulating microelectrode placed directly in the corpus callosum.

DA has been reported to decrease the release of glutamate from neostriatal slices (Mitchell and Doggett, 1980; Rowlands and Roberts, 1980) and receptor binding studies have suggested that the terminals of neocortical afferents possess DA receptors (Schwarcz et al., 1978). However, we found that bath application of DA $[1-100 \mu \mathrm{M}$ with $(n=8)$ or without $(n=7) 0.3 \mathrm{mM}$
A CONTROL
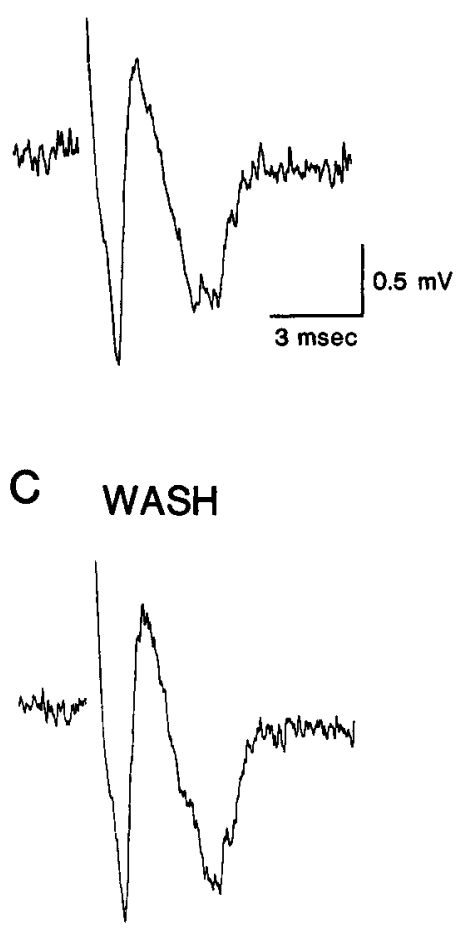

\section{KYNURENATE}

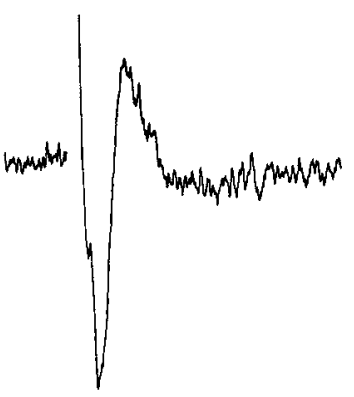

Figure 3. Dopamine has no effect on the locally evoked field potential. $\Lambda-C, \Lambda$ pplication of $\mathrm{D} \Lambda(30 \mu \mathrm{M})$ had no significant effect on either N1 or $\mathrm{N} 2$. All solutions contained $0.3 \mathrm{~mm}$ ascorbate. $D$, Application of kynurenate (1 mM) to the same field potential significantly reduced $\mathrm{N} 2$.

ascorbate; Fig. 3] had no consistent or significant effects on either $\mathrm{N} 1$ or N2. This lack of effect of DA on N2 is consistent with the results of 2 recent studies, which call into question the existence of DA receptors on the terminals of corticostriatal afferents (Trugman et al., 1986; Joyce and Marshall, 1987).

In contrast to $\mathrm{DA}$, muscarinic agonists have been reported to significantly reduce $\mathrm{N} 2$ and intracellularly recorded EPSPs (Takagi and Yamamoto, 1978; Misgeld et al., 1982; Weiler et al., 1984). In agreement with these reports, we found that carbachol (5-20 $\mu \mathrm{M})$, a muscarinic agonist, reversibly reduced or blocked N2 (Figs. 4, $5 ; n=13$ ). Carbachol's action was prevented by the coapplication of atropine (1-3 $\mu \mathrm{M}$ ) (Fig. $4 E ; n=7$ ), demonstrating that carbachol was indeed acting on muscarinic receptors in the striatum. Figure $4 F$ also demonstrates that the same $\mathrm{N} 2$ component that was reduced by carbachol was also eliminated by kynurenate. Contrary to previous reports (Misgeld et al., 1982; Weiler et al., 1984), application of atropine by itself did not increase the amplitude of N2 (Fig. $4 D$ ), even when the N2 amplitude was clearly submaximal.

To begin to test whether carbachol was exerting its actions pre- or postsynaptically, the response to ionophoretically applied glutamate was recorded while simultaneously recording the synaptically evoked field potential from the same location. If an agent is acting presynaptically and inhibiting transmitter release, then the ionophoretically induced glutamate field potential should be unaffected at a time when the synaptically mediated field is reduced. An agent acting postsynaptically would be expected to affect both field potentials to a similar degree (sec Discussion for possible problems with this interpretation).

Figure 5 shows that the ionophoretically induced glutamate 
A

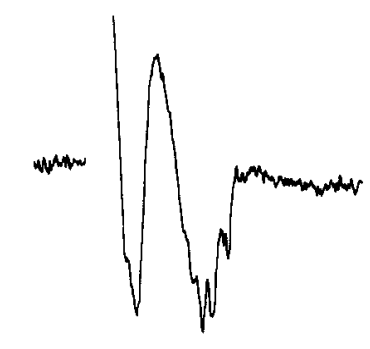

D ATROPINE

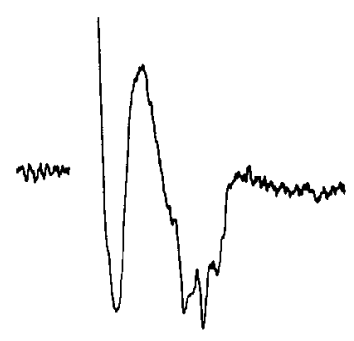

B CARBACHOL

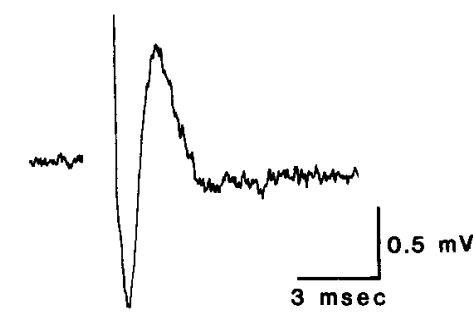

E ATRO+CARB

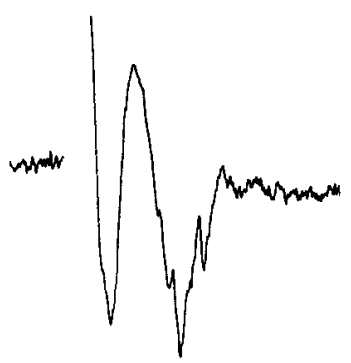

C WASH

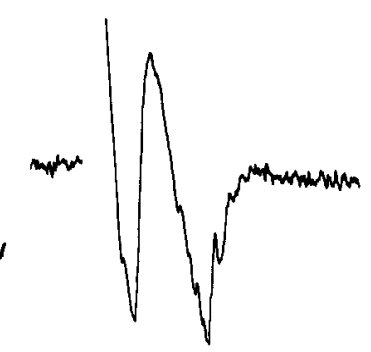

F KYNURENATE

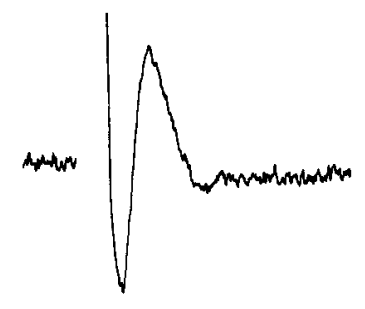

Figure 4. Muscarinic receptor activation reduces N2. $A-C$, Application of carbachol $(10 \mu \mathrm{M})$ reversibly reduced N2 without affecting N1. $D, E$. Subsequent application of atropine $(2 \mu \mathrm{M})$ had no effect on the field by itself, but blocked the action of carbachol. $F$, After washout of carbachol and atropine, kynurenate (1 mM) blocked N2. field potential is unaffected by carbachol at a time when the stimulus-evoked N2 component is significantly reduced. Kynurenate, unlike carbachol, reduced both field potentials simultaneously with a similar time course, demonstrating that the field potential recorded in response to ionophoretic application of glutamate was indeed due to the activation of functional glutamate receptors. These results are consistent with the hypothesis that muscarinic receptors localized on the terminals of corticostriatal afferents can inhibit transmitter release.

Adenosine has been found to presynaptically inhibit excitatory synaptic transmission in a variety of brain regions (Okada and Ozawa, 1980; Kocsis et al., 1984; Dunwiddie and Haas, 1985). Both in situ radioligand binding studies (Goodman and Snyder, 1982) and electrophysiological studies (Kostopoulos and Phillis, 1977; Trussell and Jackson, 1985) suggest that there are moderately high concentrations of adenosine receptors in the neostriatum. Furthermore, adenosine antagonists have locomotor stimulatory effects, suggesting a rolc for adcnosine in the control of motor activity (Daly et al., 1981). Therefore, we examined the actions of adenosine on $\mathrm{N} 1$ and N2. Like kynurenate and carbachol, adenosine $(0.1-0.5 \mathrm{~mm})$ reversibly decreased or abolished N2 without significantly affecting N1 (Fig. 6, $A-C ; n$ $=8$ ). Application of theophylline (0.1-0.3 mM), a well-established adenosine antagonist (Daly et al., 1981), blocked the reduction of $\mathrm{N} 2$ by adenosine (Fig. $6 E ; n=3$ ), demonstrating that this action is mediated by adenosine receptors.

To determine whether adenosine was acting primarily pre- or postsynaptically to reduce $\mathrm{N} 2$, we again utilized the procedure of recording simultaneously the synaptically evoked N2 and the field response to ionophoretically applied glutamate. Figure $7 B$ shows that at a time when adenosine had significantly reduced $\mathrm{N} 2$, the glutamate field potential was unaltered $(n=3)$. In contrast, kynurenate reduced both field potentials simultaneously (Fig. $7 D$ ). Thus, like carbachol, adenosine likely reduces or blocks $\mathrm{N} 2$ by acting presynaptically to inhibit transmitter release.

\section{Discussion}

The corticostriatal projection comprises the major input to the neostriatum using glutamate as the neurotransmitter (Dray, 1980; Graybiel and Ragsdale, 1983). In agreement with previous suggestions (Takagi and Yamamoto, 1978; Cordingley and Weight, 1986), we have presented evidence that the second negativity (N2) of the locally evoked field potential in neostriatal slices corresponds primarily to the synaptic activation of striatal neurons by cortical afferents. Thus, 3 different excitatory amino acid antagonists (kynurenate, piperidine dicarboxylate, and $\gamma$-Dglutamylglycine) reversibly reduce or block N2. Furthermore, direct stimulation of the corpus callosum also elicits a kynurenate-sensitive N2 component of the field potential.

Anatomical studies have demonstrated that the only neostriatal afferents that run through the corpus callosum come from neocortex (Carman et al., 1965; Carpenter and Sutin, 1983). Thalamic afferents pass dircetly into the ncostriatum (Powell and Cowan, 1956; Carpenter and Sutin, 1983) and would not be activated by callosal stimulation. There is no evidence to support the existence of intrinsic glutaminergic neurons or afferent sources of excitatory amino acids other than cerebral cortex and thalamus. Afferent or intrinsic pathways not utilizing excitatory amino acid transmitters are also likely stimulated when the cortical afferents are activated, but the virtual absolute blockade of $\mathrm{N} 2$ by kynurenate strongly suggests that $\mathrm{N} 2$ overwhelmingly reflects the synaptic responses of neostriatal neurons to the activation of cortical afferents.

Misgeld and colleagues (1980) have previously presented evidence that nicotinic antagonists can reduce $\mathrm{N} 2$. However using the same nicotinic antagonists as Misgeld et al. (1980), we found no effect on $\mathrm{N} 2$. We have no explanation for this discrepancy, except to note that neither Cordingley and Weight (1986) nor Takagi and Yamamoto (1978) found that nicotinic antagonists had any significant actions on N2. 
A CONTROL

1

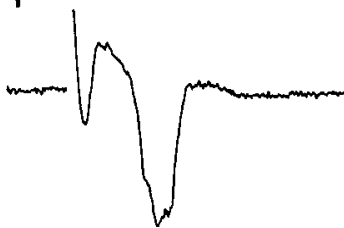

2 gLutAMATE

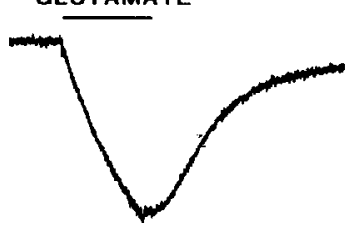

C WASH

1

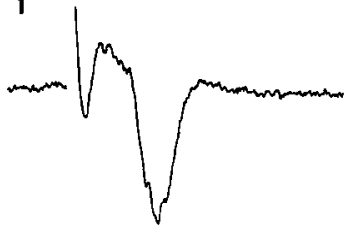

2

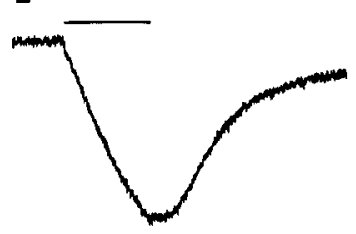

B CARBACHOL

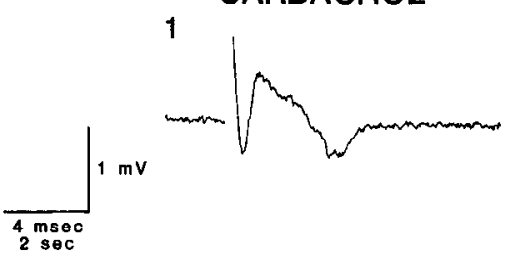

2

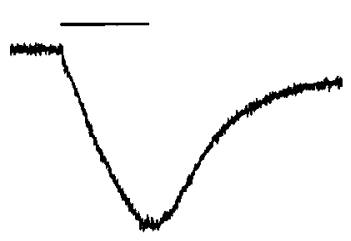

D KYNURENATE 1

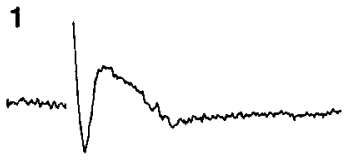

Muscarinic agonists have consistently been found to reduce N2 (Takagi and Yamamoto, 1978; Weiler et al., 1984), as well as intracellularly recorded EPSPs (Dodt and Misgeld, 1986), but also have significant direct depolarizing actions on striatal neurons (Dodt and Misgeld, 1986). Using the technique of simultaneously recording the synaptically evoked N2 and the field potential evoked by the ionophoretic application of glutamate, we found that while both responses were reduced by kynurenate, only N2 was affected by carbachol. We interpret this result as indicating that carbachol is acting presynaptically to inhibit corticostriatal synaptic transmission. Further support for this hypothesis comes from the observation that carbachol, at the doses used in this study, has no effect on either the membrane potential or input resistance of striatal neurons (Dodt and Misgeld, 1986).

$\mathrm{ACh}$, acting on muscarinic receptors, has been shown to inhibit synaptic transmission at synapses utilizing excitatory amino acids in a variety of systems, including rat ventral horn cells (Jiang and Dun, 1986) and hippocampus (Hounsgaard, 1978; Valentino and Dingledine, 1981). The presynaptic inhibitory action of $\mathrm{ACh}$ may be due to either a reduction in calcium conductance or to an increase in potassium conductance occurring at or near the axon terminal (North, 1986).

The validity of our conclusion concerning the locus of car-

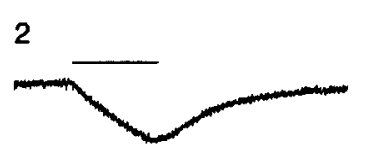

Figure 5. Carbachol acts presynaptically to reduce N2. The field potential in response to electrical stimulation $(I)$ and the response to ionophoretic application of glutamate (2) recorded from the same location are shown following carbachol and kynurenate application. $A-$ $C$, Carbachol $(10 \mu \mathrm{M})$ reversibly reduced N2 without affecting the glutamate response, while application of kynurenate $(D)$ to the same fields reduced both simultaneously.
A CONTROL

\section{THEOPHYLLINE}

Figure 6. Adenosine receptor activation reduces N2. $A-C$, Application of adenosine $(0.2 \mathrm{~mm})$ reversibly reduced N2 without altering N1. $D, E$, Theophylline $(0.1 \mathrm{~mm})$ by itself had no effect on the field but inhibited the action of adenosine, demonstrating that adenosine was acting at adenosine receptors. $F$, N2 was subsequently blocked by piperidine dicarboxylate $(P D A ; 1.0 \mathrm{~mm})$, an excitatory amino acid antagonist, again demonstrating that $\mathrm{N} 2$ is mediated by a glutamate-like compound.

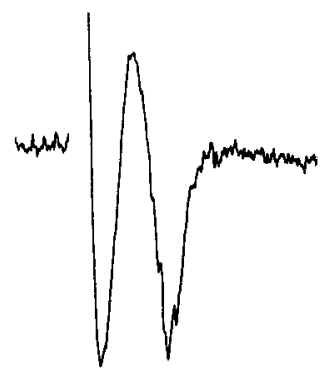

B ADENOSINE

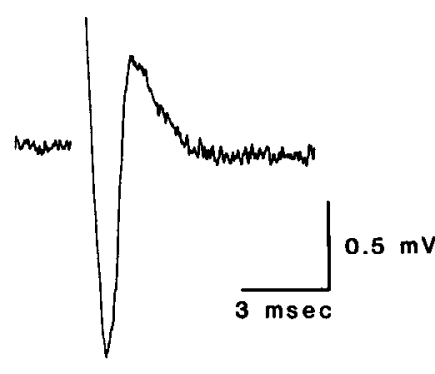

E ADEN+THEOPH

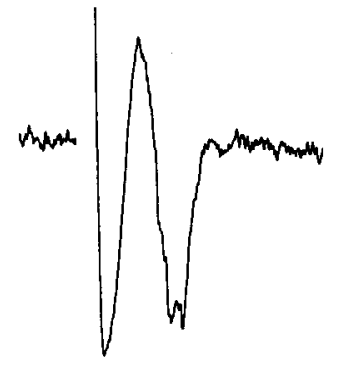

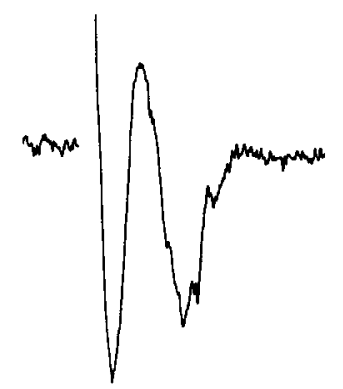

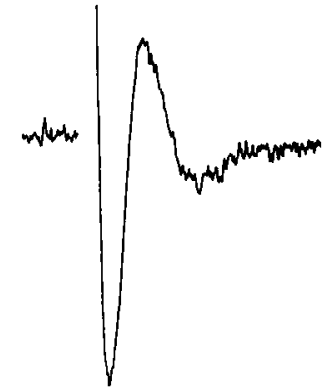



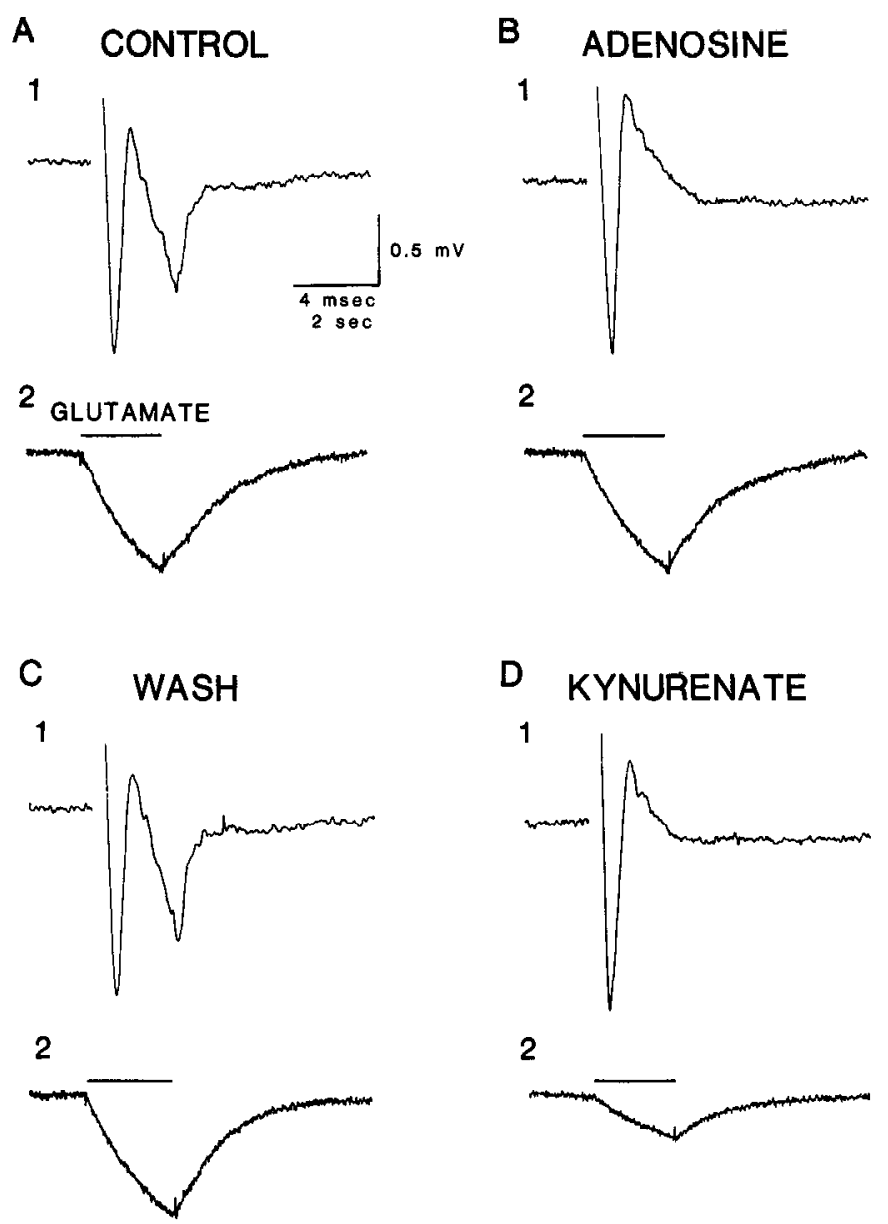

Figure 7. Adenosine acts presynaptically to inhibit corticostriatal synaptic transmission. $A-C$, Adenosine reversibly reduced N2 (I), while the simultaneously recorded field potential in response to ionophoretic application of glutamate (2) was unaffected. $D$, In contrast, kynurenate ( $1 \mathrm{~mm}$ ), applied to the same slice, reduced both $\mathrm{N} 2$ and the field potential in response to glutamate application.

bachol's action is dependent on the assumption that the same group of postsynaptic glutamate receptors is being activated by synaptically released transmitter and the exogenously applied glutamate. This seems likely, since both responses were recorded via the same recording electrode, but it is conceivable that the 2 responses reflect the activation of different populations of receptors, with different sensitivities to the postsynaptic actions of carbachol.

The biochemical anatomy of the neostriatum has received a great deal of attention (Graybiel and Ragsdale, 1983). One fairly well-developed classification, based primarily on staining for $\mathrm{AChE}$, divides the neostriatum into striosomes and matrix. Striosomes are areas that stain poorly for AChE (Graybiel and Ragsdale, 1978), exhibit dense patches of opiate receptor binding (Herkenham and Pert, 1981), and are enriched in immunoreactivity for enkephalin and substance $P$ (Graybiel et ai.., 1981). The extrastriosomal matrix exhibits high AChE histochemical staining (Graybiel and Ragsdale, 1978) and high immunoreactivity to choline acetyltrasferase (Graybiel et al., 1986), suggesting that the cholinergic neuropil is concentrated primarily in the matrix. In our experiments, carbachol always reduced N2 independent of microelectrode placement. This may reflect (1) the fact that the striosomal-matrix distinction becomes much less dramatic in adult animals (Graybiel and Ragsdale, 1983), (2) the inability of our recording techniques to discern subtle spatial differences in the density of muscarinic receptors present in neostriatum, or (3) our selection criteria for "good" field potentials inadvertently results in the consistent placement of the recording electrode in the matrix.

Like carbachol, adenosine reduced or blocked N2 at a time when the glutamate field response was unaffected, suggesting that adenosine may also presynaptically inhibit synaptic transmission between cortical afferents and neostriatum. Adenosine has a similar action in the hippocampus, where it has been shown to inhibit the release of glutamate (Dolphin and Archer, 1983; Corradetti et al., 1984) and to presynaptically inhibit excitatory synaptic transmission (Okada and Ozawa, 1980; Dunwiddie and Haas, 1985). Functional adenosine receptors are also found on the terminals of the cerebellar parallel fibers (Kocsis et al., 1984). In cultured neostriatal cells, adenosine has been shown to increase membrane conductance to potassium (Trussell and Jackson, 1985). If adenosine had the same action on presynaptic nerve terminals, it would be expected to decrease stimulus-evoked neurotransmitter release. However, adenosine may also have actions on the voltage-dependent calcium conductance at the terminal (Wakade and Wakade, 1978) or interfere intracellularly with the calcium-stimulated secretory process (Silinsky, 1984).

All areas of the cerebral cortex project to the striatum topographically. However, the corticostriatal projection is not uniform, but derives, rather, from a number of distinct subclasses of cortical neurons with different axonal collaterals and projections (Jones et al., 1977; Wilson, 1987). Furthermore, diverse cortical regions may project to the same striatal zones (Selemon and Goldman-Rakic, 1985; Malach and Graybiel, 1986). The segregation of receptors for ACh or adenosine on distinct subsets of corticostriatal fibers would permit the selective inhibition of input to the striatum from diverse regions of cerebral cortex or from distinct classes of cortical neurons. Quantitative autoradiography of binding sites following selective cortical lesions will help determine whether all or a subset of corticostriatal fibers possess receptors for ACh or adenosine.

\section{References}

Carman, J. B., W. M. Cowan, T. P. S. Powell, and K. E. Webster (1965) A bilateral cortico-striate projection. J. Neurol. Neurosurg. Psychiatry 28: $71-77$.

Carpenter, M. B., and J. Sutin (1983) Human Neuroanatomy, 8th Ed., pp. 587-589, Williams \& Wilkins, Baltimore, MD.

Cordingley, G. E., and F. F. Weight (1986) Non-cholinergic synaptic excitation in neostriatum: Pharmacological evidence for mediation by a glutamate-like transmitter. Br. J. Pharmacol. 88: 847-856.

Corradetti, R., G. Moneti, F. Moroni, G. Pepeu, and A. Wieraszko (1984) Adenosine decreases aspartate and glutamate release from rat hippocampal slices. Eur. J. Pharmacol. 104: 19-26.

Daly, J. W., R. F. Bruns, and S. H. Snyder (1981) Adenosine receptors in the central nervous system: Relationship to the central actions of methylxanthines. Life Sci. 28: 2083-2097.

Dodt, H. U., and U. Misgeld (1986) Muscarinic slow excitation and muscarinic inhibition of synaptic transmission in the rat neostriatum. J. Physiol. (Lond.) 380: 593-608.

Dolphin, A. C., and E. R. Archer (1983) An adenosine agonist inhibits and a cyclic AMP analogue enhances the release of glutamate but not GABA from slices of rat dentate gyrus. Neurosci. Lett. 43: 49-54.

Dray, A. (1980) The physiology and pharmacology of mammalian basal ganglia. Prog. Neurobiol. 14: 221-335.

Dunwiddie, T. V., and H. L. Haas (1985) Adenosine increases synaptic facilitation in the in vitro rat hippocampus: Evidence for a presynaptic site of action. J. Physiol. (Lond.) 369: 365-377. 
Ganong, A. H, T. H. Lanthorn, and C. W. Cotman (1983) Kynurenic acid inhibits synaptic and acidic amino acid-induced responses in the rat hippocampus and spinal cord. Brain Res. 273: 170-174.

Ginsborg, B. L., and G. D. S. Hirst (1972) The effect of adenosine on the release of the transmitter from the phrenic nerve of the rat. $J$. Physiol. (Lond.) 224: 629-645.

Goodman, R. F., and S. H. Snyder (1982) Autoradiographic localization of adenosine receptors in rat brain using $\left[{ }^{3} \mathrm{H}\right]$ cyclohexyladenosine. J. Neurosci. 2: 1230-1241.

Graybiel, A. M., and C. W. Ragsdale, Jr. (1978) Histochemically distinct compartments in the striatum of human, monkey, and cat demonstrated by acetycholinesterase staining. Proc. Natl. Acad. Sci. USA 75: 5723-5726.

Graybiel, A. M., and C. W. Ragsdale, Jr. (1983) Biochemical anatomy of the striatum. In Chemical Neuroanatomy, P. C. Emson, ed., pp. 427-504, Raven, New York.

Graybiel, A. M., C. W. Ragsdale, Jr., E. S. Yoneoka, and R. P. Elde (1981) An immunohistochemical study of cnkephalins and other neuropeptides in the striatum of the cat with evidence that the opiate peptides are arranged to form mosaic patterns in register with the striosomal compartments visible by acetylcholinesterase staining. Neuroscience 6: 377-397.

Graybiel, A. M., R. W. Baughman, and F. Eckenstein (1986) Cholinergic neuropil of the striatum observes striosomal boundaries. Nature 323: $625-627$

Herkenham, M., and C. B. Pert (1981) Mosaic distribution of opiate receptors, parafascicular projections and acetylcholinesterase in rat striatum. Nature 291: 415-418.

Hounsgaard, J. (1978) Presynaptic inhibitory action of acetycholine in area CA1 or the hippocampus. Exp. Neurol. 62: 787-797.

Jahr, C. E., and K. Yoshioka (1986) Ia afferent excitation of motoncurones in the in vitro new-born rat spinal cord is sclcctivcly antagonized by kynurenate. J. Physiol. (Lond.) 370: 515-530.

Jiang, Z. G., and N. J. Dun (1986) Presynaptic suppression of excitatory postsynaptic potentials in rat ventral horn neurons by muscarinic agonists. Brain Res. 381: 182-186.

Jones, E. G., J. D. Coulter, H. Burton, and R. Porter (1977) Cells of origin and terminal distribution of corticostriatal fibers arising in the sensory-motor cortex of monkeys. J. Comp. Neurol. 173: 53-80.

Joyce, J. N., and J. F. Marshall (1987) Quantitative autoradiography of dopamine $\mathrm{D}_{2}$ sites in rat caudate-putamen: Localization to intrinsic neurons and not to neocortical afferents. Neuroscience $20: 773-795$.

Kemp, J. M., and T. P. S. Powell (1971) The site of termination of afferent fibers in the caudate nucleus. Phil. Trans. R. Soc. Lond. [Biol.] 262: $413-427$

Kita, H., T. Kita, and S. T. Kitai (1985) Active membrane properties of rat neostriatal neurons in an in vitro slice preparation. Exp. Brain Res. 60: 54-62.

Kocsis, J. D., D. L. Eng, and R. B. Bhisitkul (1984) Adenosine selectively blocks parallel-fiber-mediated synaptic potentials in rat cerebellar cortex. Proc. Natl. Acad. Sci. USA 81: 6531-6534.

Kornhuber, J., and M. E. Kornhuber (1986) Presynaptic dopaminergic modulation of cortical input to the striatum. Life Sci. 39: 669-674.

Kostopoulos, G. K., and J. W. Phillis (1977) Purinergic depression of neurons in different areas of the rat brain. Exp. Neurol. 55:719-724.
Malach, R., and A. M. Graybiel (1986) Mosaic architecture of the somatic sensory-recipient sector of the cat's striatum. J. Neurosci. 6 : $3436-3458$.

Misgcld, U., X. Okada, and R. Hassler (1979) Locally evoked potentiàs in slices of rat neostriatum: A tool for the investigation of intrinsic excitatory processes. Exp. Brain Res. $34: 575=590$.

Misgeld, U., M, H, Weiler, and I. J. Bak (1980) Intrinsic cholinergic excitation in the rat neostriatum: Nicotinic and muscarinic receptors. Exp. Brain Res. 39: 401-409.

Misgeld, U., M. H. Weiler, and D. K. Cheong (1982) Atropine enhances nicotinic cholinergic EPSPs in rat neostriatal slices. Brain Res. 253: $317-320$.

Mitchell, P. R., and N. S. Doggett (1980) Modulation of striatal [ $\left.{ }^{3} \mathrm{H}\right]-$ glutamic acid release by dopaminergic drugs. Life Sci. 26: 2073-2081.

North, R. A. (1986) Muscarinic receptors and membrane ion conductances. Trends Pharmacol. Sci. (Suppl.) 7: 19-22.

Okada, Y., and S. Ozawa (1980) Inhibitory action of adenosine on synaptic transmission in the hippocampus of the guinea pig in vitro. Eur. J. Pharmacol. 68: 483-492.

Powell, T. P. S., and W. M. Cowan (1956) A study of thalamo-striate relations in the monkey. Brain 79: 364-390.

Rowlands, G. J., and P. J. Roberts (1980) Activation of dopaminergic receptors inhibits calcium-dependent glutamate release from corticostriatal terminals in vitro. Eur. J. Pharmacol. 62: 241-242.

Schwarcz, R., I. Creese, J. T. Coyle, and S. H. Snyder (1978) Dopamine receptors localized on cerebral cortical afferents to rat corpus striatum. Nature 271: 766-768.

Selemon, L. D., and P. S. Goldman-Rakic (1985) Longitudinal topography and interdigitation of corticostriatal projections in the rhesus monkey. J. Neurosci. 5: 776-794.

Silinsky, E. M. (1984) On the mechanism by which adenosine receptor activation inhibits the release of acetylcholine from motor nerve endings. J. Physiol. (Lond.) 346: 243-256.

Takagi, M., and C. Yamamoto (1978) Suppressing action of cholinergic agents on synaptic transmissions in the corpus striatum of rats. Exp. Neurol. 62: 433-443.

Trugman, J. M., W. A. Geary, II, and G. F. Wooten (1986) Localization of D-2 dopamine receptors to intrinsic striatal neurones by quantitative autoradiography. Nature 323: 267-269.

Trussell, L. O., and M. B. Jackson (1985) Adenosine-activated potassium conductance in cultured striatal neurons. Proc. Natl. Acad. Sci. USA $82: 4857-4861$.

Valentino, R. J., and R. Dingledine (1981) Presynaptic inhibitory effect of acetylcholine in the hippocampus. J. Neurosci. 1: 784-792.

Wakade, A. R., and T. D. Wakade (1978) Inhibition of noradrenaline release by adenosine. J. Physiol. (Lond.) 282: 35-49.

Weiler M. H., U. Misgeld, and D. K. Cheong (1984) Presynaptic muscarinic modulation of nicotinic excitation in the neostriatum. Brain Res. 296: 111-120.

Wilson, C. J. (1987) Morphology and synaptic connections of crossed corticostriatal neurons in the rat. J. Comp. Neurol. 263: 567-580.

Yamamoto, C. (1973) Neurotransmitter actions studied in brain slices. Adv. Neurol. Sci. 17: 64-70. 